\title{
Comparación morfológica entre el útero fetal y el útero adulto de la alpaca (Vicugna pacos) y la llama (Lama glama)
}

Morphologic comparison between fetal uterus and adult uterus in the alpaca (Vicugna pacos) and the llama (Lama glama)

GalyMendoza $^{1}$, Luisa Echevarría ${ }^{1}$, Cielo Llerena ${ }^{1},{\text { Alejandra } \text { Castro }^{2} \text {, María Domínguez }}^{2}$, Susana Gómez ${ }^{2}$, Marcelo Ghezzi ${ }^{2}$, Claudio Barbeito ${ }^{3}$

\section{RESUMEN}

Objetivos: Comparar los aspectos morfológicos del tracto genital fetal y adulto de la alpaca y la llama. Material y métodos: se realizó el estudio anatómico del útero de cinco fetos de llama y cinco fetos de alpaca, ambos de tercer tercio de gestación, once úteros de alpacas adultas y cinco úteros de llamasadultas. Las muestras de útero se estudiaron por observación anatómica y morfometría. Resultados: Mediante los estudios morfométricos se pudo observar que existía diferencia significativa en el tamaño de ambos cuernos uterinosa favor del lado izquierdo. La relación existente entre el cuerno uterino derecho y el cuerno uterino izquierdo en la alpaca adulta es de 0,79 y en la llama adulta la razón de las medidas es mayor, 0,85 para la longitud y 0,87 para el ancho. En los úteros fetales tanto en llama como en alpaca, se observó que existía diferencia significativa entre el tamaño del cuerno uterino izquierdo y el cuerno uterino derecho a favor del lado izquierdo. Conclusiones: Se concluye que existe similitud entre la forma del útero en los fetos de llama y alpaca en relación al útero de la correspondiente hembra adulta.

PALABRAS CLAVE:Útero, morfometría, alpaca, llama.

\section{SUMMARY}

Objectives: Made a morphologic comparison between fetal uterus and adult uterus in the alpaca and the llama. Material and methods: it was performed anatomical study of five fetal uteri of lama and alpaca, eleven adult alpacas and five lamas uteri. Uterus samples were studied by anatomical observation and morphometry. Results: The results showed that all the samples of adult females had greater development of the left uterine horn. By morphometric studies it was observed that there was significant difference between the size of both uterine horns. The relationship between the right uterine horn and left uterine horn in the alpaca is 0.79 and in the lama, the ratio of the measures is higher, 0.85 for length and 0.87 to the width, in favor of the left uterine horn. In both fetal uterus in the lama as alpaca, was observed that there was significant difference between the size of the left uterine horn and the right uterine horn. Conclusions: exits similarity between the shape of the uterus in the lama and alpaca fetuses in relation to the uterus of the corresponding adult female.

KEYWORDS:Uterus, morphometry, alpaca, llama.

${ }^{1}$ Facultad de Medicina Veterinaria y Zootecnia, Universidad Peruana Cayetano Heredia. Lima, Perú.

${ }^{2}$ Facultad de Ciencias Veterinarias, Universidad Nacional del Centro de la Provincia de Buenos Aires. Buenos Aires, Argentina.

${ }^{3}$ Facultad de Ciencias Veterinarias, Universidad Nacional de La Plata. La Plata, Buenos Aires. CONICET. Argentina. 


\section{INTRODUCCIÓN}

Los Camélidos Sudamericanos, también conocidos como Camélidos del Nuevo Mundo, incluyen a la llama (Lama glama) y la alpaca (Vicugna pacos) como especies domésticas y al guanaco (Lama guanicoe) y la vicuña (Vicugnavicugna) como especies silvestres. Estos animales forman parte de la actividad pecuaria en las zonas alto andinas de países como Perú, Argentina, Chile y Bolivia. Su importancia económica radica en la explotación de su fibra y su capacidad de transformar productos agrícolas en alimento para consumo humano. Su crianza ha aumentado en Sudamérica e incluso han sido llevadas algunas especies a otros continentes donde su capacidad de adaptación a diversos medio ambientes ha permitido su producción y la mejora genética.

Se han realizado numerosos trabajos de investigación sobre estas especies: Si bien su biología reproductiva ha sido muy estudiada, existe menos información sobre las características anatómicas de los órganos genitales, en comparación con las fisiológicas del tracto reproductor de las hembras, que constituyen el principal punto de atención de los investigadores.

El tracto genital hembra de los Camélidos Sudamericanos incluye: dos ovarios, dos oviductos, un útero conformado por dos cuernos uterinos confluyentes al cuerpo y seguido del cuello, la vagina y la vulva $(1,2)$; estos órganos también forman el sistema genital de la camella (3), la cerda, la yegua, la vaca y la oveja (4), entre otras especies.

La alpaca y la llama son especies de ovulación inducida. La ovulación puede ser inducida por la monta o cópula y por la aplicación parenteral de HCG o de GnRH; se ha encontrado, además, que existe ovulación espontánea sin causa aparente que la provoque (5). La bolsa ovárica envuelve por completo al ovario y así facilita el paso del óvulo hacia el oviducto, donde se fertiliza y se mantiene alrededor de 3 a 6 días después de la cópula. Cuando el embrión llega al útero, generalmente se implanta en el cuerno uterino izquierdo en un $95 \%$ a $98,4 \%$ de las veces $(6,7)$.

En apariencia, el cuerno uterino izquierdo es de mayor tamaño que el cuerno uterino derecho (8). Estas características también se repiten en el camello y en el dromedario (9). Del Campo en 1996 encontró que esta diferencia de tamaño de los cuernos se ha observado además en fetos de alpacas y llamas(10).
En base a lo anterior, el presente trabajo se realizó con el objeto de comparar la conformación del útero fetal $\mathrm{y}$ adulto de llamas y alpacas.

\section{MATERIAL Y MÉTODOS}

La toma de muestras se realizó en el Camal Municipal de la Ciudad de Huancavelica y en el Camal Municipal de Pilpichaca, Departamento de Huancavelica, Perú. Las llamas y alpacas se seleccionaron al azar al llegar al camal para su sacrificio, su edad oscilaba entre los 2 a 14 años de edad. La condición corporal estuvo entre grado 3 y 4 según la escala de la Australian Alpaca Association, 2008. El peso corporal osciló entre 50 a 60 kilogramos para las alpacas y 80 a 90 kilogramos para las llamas.

Se recolectaron 11 úteros no gestantes de alpacas y 5 similares de llamas, todas adultas, además, 5 úteros de fetos de alpaca y 5 úteros de fetos de llama, todos estos fetos fueron del tercer tercio de gestación. Se realizó el cálculo de la edad gestacional utilizando una fórmula, basada enla medición del diámetro biparietal (Figura 1), las edades fluctuaron entre los 295 a los 330 días.

El análisis de las muestras se realizó en el laboratorio de anatomía animal de la Facultad de Medicina Veterinaria (FAVEZ) y Zootecnia de la Universidad Peruana Cayetano Heredia, Lima, Perú y el laboratorio de Anatomía Animal de la Facultad de Ciencias Veterinarias de la Universidad Nacional del Centro de la Provincia de Buenos Aires, Tandil, Argentina.

Los úteros de los animales adultos se trasladaron refrigerados al Laboratorio de Anatomía de la FAVEZ y se mantuvieron congelados a una temperatura de -5

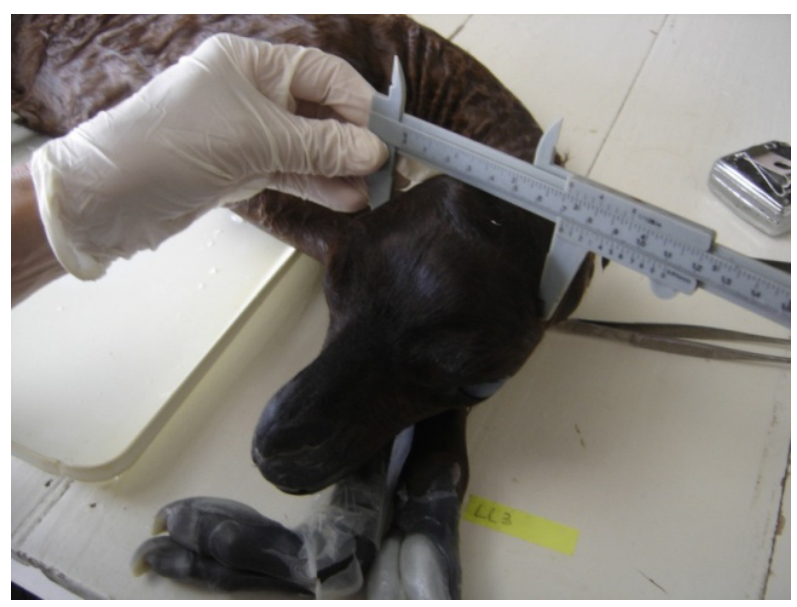

Figura 1. Medición del diámetro biparietal 
${ }^{\circ} \mathrm{C}$ hasta su procesamiento. Para su descongelamiento se colocaron a temperatura ambiente por seis horas y luego se sumergieron en suero fisiológico tibio. Se pesaron los úteros en una balanza digital ${ }^{\circledR}$ Ishida. Los úteros no gestantes se clasificaron según el estado fisiológico de los ovarios en tres grupos; con folículos en crecimiento, con folículos maduros (mayor de 7 $\mathrm{mm}$ de diámetro) y con cuerpo lúteo (Figura 2). Se asumió que aquellos que presentaban cuerpo lúteo fueron de casos de ovulación espontánea.

Los fetos fueron fijados utilizando para ello una solución de formol al 10\%, que fue aplicada por vía intraperitoneal. El abordaje a la cavidad se realizó a nivel del abdomen caudal, mediante un corte desde la raíz umbilical hacia el borde craneal del pubis, seguido de dos cortes laterales a nivel de la región inguinal por el borde craneal del muslo. Se disecaron los planos musculares, la fascia transversa y el peritoneo parietal.

Se registraron los datos en planillas de Excel y tablas en Word, las imágenes digitales incluían una regla en

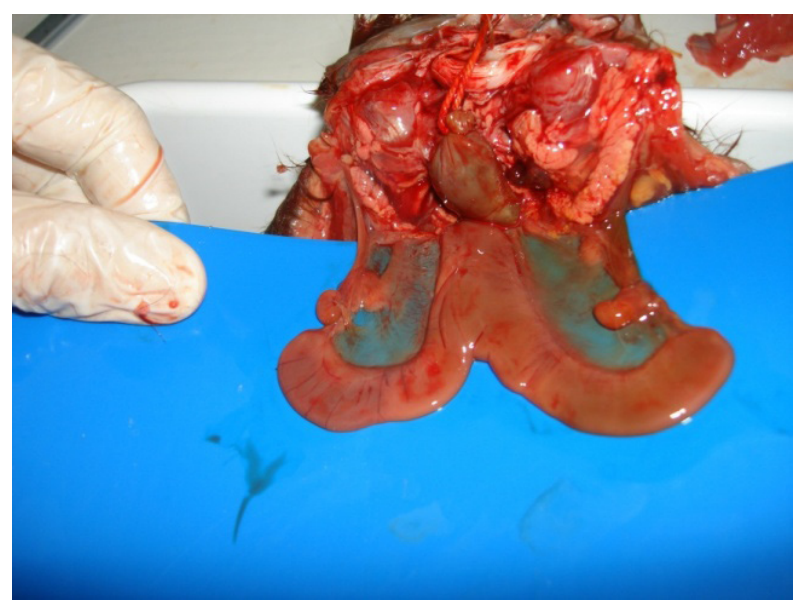

Figura 2. Observación del útero in situ la fotografía para facilitar el estudio morfométrico correspondiente. Los términos anatómicos utilizados fueron los correspondientes a la Nómina Anatómica, Histológica y Embriológica Veterinaria (13). Las imágenes obtenidas fueron procesadas mediante el uso del sistema para morfometríaLeica Microsystems ${ }^{\circledR}$ LeicaQWinimageprocessing and analysisworkstation V2.6., Leica Microsystems ImagingSolutionsLtd, Cambridge, UK.Los resultados fueron presentados mediante estadística descriptiva paramétrica (media, desvío estándar). Se utilizó el software estadístico SPSS.

\section{RESULTADOS}

El estudio anatómico de los úteros en adultos y fetos,evidencióque los cuernos uterinos al extenderse adoptan una distribución cónica, con un extremo angosto relacionado al oviducto y un extremo ancho relacionado al cuerpo del útero. Los cuernos uterinos se observaron con una distribución espiraladay una ligera torsión, adoptando una posición lateral luego de girar hacia caudal y ventral. Cada cuerno uterino se relaciona con el respectivo oviducto y ovario.

El estudio morfométrico realizado para evaluar los cuernos uterinos de las hembras adultas, mostró diferencia significativa a favor del tamaño del cuerno uterino izquierdo, tanto en la alpaca como en la llama (Tabla 1). Al evaluar la relación existente entre el tamaño del cuerno uterino derecho y el cuerno uterino izquierdo en la alpaca, esta fue de 0.79 (aproximadamente $80 \%$ ), este valor varía según el estado fisiológico del órgano, siendo mayor al momento en que los ovarios presentan cuerpo lúteo. En la llama, la razón de las medidas es mayor (0.85 para la longitud y 0.87 para el ancho) (Tabla 2).

Tabla 1. Medidas (cm) de los cuernos uterinos de alpacas $(\mathrm{n}=11)$ y llamas $(n=5)$.

\begin{tabular}{cccccc}
\hline \multirow{2}{*}{ Especie } & Medidas & \multicolumn{2}{c}{ Lado Derecho } & \multicolumn{2}{c}{ Lado Izquierdo } \\
\cline { 3 - 6 } & & Media & $\begin{array}{c}\text { Desviación } \\
\text { estándar }\end{array}$ & Media & $\begin{array}{c}\text { Desviación } \\
\text { estándar }\end{array}$ \\
\hline Alpaca & Longitud & $8,32^{\mathrm{a}}$ & 2,37 & $10,45^{\mathrm{b}}$ & 2,70 \\
$(\mathrm{n}=11)$ & Ancho & $3,08^{\mathrm{a}}$ & 0,54 & $3,55^{\mathrm{a}}$ & 0,77 \\
$\begin{array}{c}\text { Llama } \\
(\mathrm{n}=5)\end{array}$ & Longitud & $10,78^{\mathrm{a}}$ & 2,26 & $12,70^{\mathrm{a}}$ & 2,80 \\
& Ancho & $3,92^{\mathrm{a}}$ & 0,30 & $4,50^{\mathrm{b}}$ & 0,34 \\
\hline
\end{tabular}

a,b Letras diferentes indican que las medias son significativamente diferentes según fue determinado por la prueba de T de Student, $\mathrm{P}<0,05$. 


\section{INVESTIGACIÓN ORIGINAL I ORIGINAL RESEARCH}

Tabla 2. Relación entre el tamaño Tiene unidad si es relación? del cuerno uterino derecho y cuerno uterino izquierdo en úteros vacíos de alpacas.

\begin{tabular}{lcc}
\hline $\begin{array}{c}\text { Según el estado fisiológico } \\
\text { reproductivo del ovario }\end{array}$ & $\begin{array}{c}\text { Longitud del cuerno } \\
\text { uterino }\end{array}$ & $\begin{array}{c}\text { Ancho del cuerno } \\
\text { uterino }\end{array}$ \\
\hline $\begin{array}{l}\text { Folículos en crecimiento, } \\
\mathrm{n}=5\end{array}$ & 0,79 & 0,87 \\
Folículos maduros, $\mathrm{n}=3$ & 0,78 & 0,91 \\
\hline Cuerpo lúteo, $\mathrm{n}=3$ & 0,80 & 0,82 \\
\hline
\end{tabular}

Tabla 3. Medidas $(\mathrm{cm})$ de los ovarios y cuernos uterinos de los fetos de alpacas $(\mathrm{n}=5)$

\begin{tabular}{cccccc}
\hline \multirow{2}{*}{ Estructura } & \multirow{2}{*}{ Medidas } & \multicolumn{2}{c}{ Lado Derecho } & \multicolumn{2}{c}{ Lado Izquierdo } \\
\cline { 3 - 5 } & & Media & Desviación estándar & Media & Desviación estándar \\
\cline { 3 - 5 } Ovario & Longitud & $5,00^{\mathrm{a}}$ & 0,135 & $5,49^{\mathrm{b}}$ & 0,394 \\
& Ancho & $3,97^{\mathrm{a}}$ & 0,097 & $4,10^{\mathrm{b}}$ & 0,079 \\
& Espesor & $3,10^{\mathrm{a}}$ & 0,106 & $2,66^{\mathrm{b}}$ & 0,305 \\
\multirow{2}{*}{ Cuerno uterino } & Longitud & $43,50^{\mathrm{a}}$ & 2,062 & $55,20^{\mathrm{b}}$ & 2,387 \\
& Ancho & $9,94^{\mathrm{a}}$ & 0,270 & $11,12^{\mathrm{b}}$ & 0,239 \\
& Espesor & $3,00^{\mathrm{a}}$ & 0,158 & $3,08^{\mathrm{a}}$ & 0,104
\end{tabular}

${ }^{a, b}$ Letras diferentes indican que las medias son significativamente diferentes $\mathrm{P}<0,05$

Tabla 4. Medidas (cm) de los ovarios y cuernos uterinos de los fetos de llamas $(\mathrm{n}=5)$

\begin{tabular}{cccccc}
\hline \multirow{2}{*}{ Estructura } & \multirow{2}{*}{ Medidas } & \multicolumn{2}{c}{ Lado Derecho } & \multicolumn{2}{c}{ Lado Izquierdo } \\
\cline { 3 - 5 } Ovario & Longitud & $6,00^{\mathrm{a}}$ & 0,127 & Media & Desviación estándar \\
\hline \multirow{2}{*}{ Cuerno uterino } & Ancho & $4,05^{\mathrm{a}}$ & 0,071 & $6,30^{\mathrm{b}}$ & 0,274 \\
& Espesor & $3,48^{\mathrm{a}}$ & 0,370 & $3,50^{\mathrm{b}}$ & 0,324 \\
& Longitud & $49,20^{\mathrm{a}}$ & 1,924 & $2,72^{\mathrm{b}}$ & 0,335 \\
& Ancho & $11,84^{\mathrm{a}}$ & 0,230 & $59,20^{\mathrm{b}}$ & 2,588 \\
& Espesor & $3,11^{\mathrm{a}}$ & 0,143 & $12,78^{\mathrm{b}}$ & 0,563 \\
\hline
\end{tabular}

${ }^{\mathrm{a}, \mathrm{b}}$ Letras diferentes indican que las medias de las medidas de los lados son significativamente diferentes $\mathrm{P}<0,05$.

El estudio anatómico topográfico de los úteros fetales en ambas especies muestra el útero, oviductos y los ovarios ubicados en el fondo de la cavidad pélvica. El tracto está sujeto a la cavidad pélvica por medio del ligamento ancho y sus componentes (mesoovario, mesosalpinx y mesometrio). El infundíbulo del oviducto envuelve por completo al ovario. A cada lado a nivel del mesometrio se observa una estructura de apariencia papilar con un pliegue propio de peritoneo que se dirige al canal inguinal. Estos órganos se relacionan en craneal con el estómago y colon transverso, hacia lateral derecho con diferentes porciones de colón ascendente y las asas yeyunales, hacia lateral izquierdo se relacionan con el colon descendente. En dorsal, el útero se relaciona con el recto, y en ventral con la vejiga urinaria, se observa además la presencia del uraco en relación al cordón umbilical. Se realizaron las mediciones de la longitud, el ancho y el espesor de los ovarios y los cuernos uterinos en los fetos de llamas y alpacas. Se observó una diferencia significativa de tamaño entre ambos cuernos uterinos a favor del cuerno uterino izquierdo (Tablas 3 y 4 ).

\section{DISCUSIÓN}

Además de las investigaciones hechas para la camella (3). Otros autores han descrito las características del tracto genital de la alpacay la llama $(1,2)$. Todos estos estudios, incluido el presente trabajo, registran la presencia de dos ovarios, dos oviductos, un útero conformado por dos cuernos, un cuerpo y un cuello, una vagina y vulva, como órganos reproductores de la alpaca y la llama (Figura 3). 


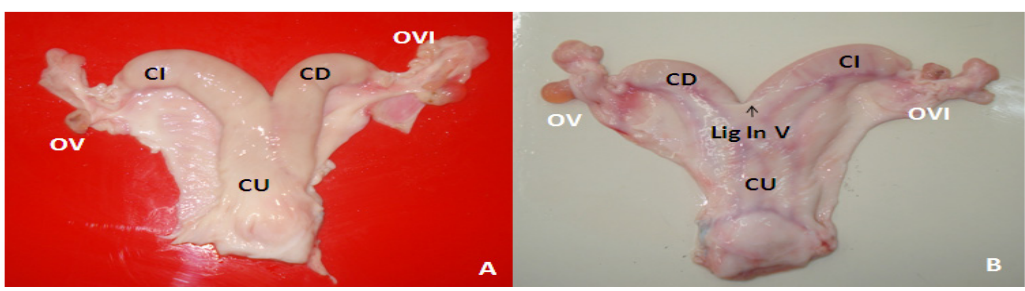

Figura 3. Utero de alpaca. A: Cara dorsal. B: Cara ventral. $\mathrm{CD}=$ cuerno uterino derecho, $\mathrm{CI}=$ cuerno uterino izquierdo, $\mathrm{CU}=$ cuerpo del útero, $\mathrm{OV}=$ ovario, $\mathrm{OVI}=$ oviducto, Lig In $\mathrm{V}=$ ligamento intercornual ventral.

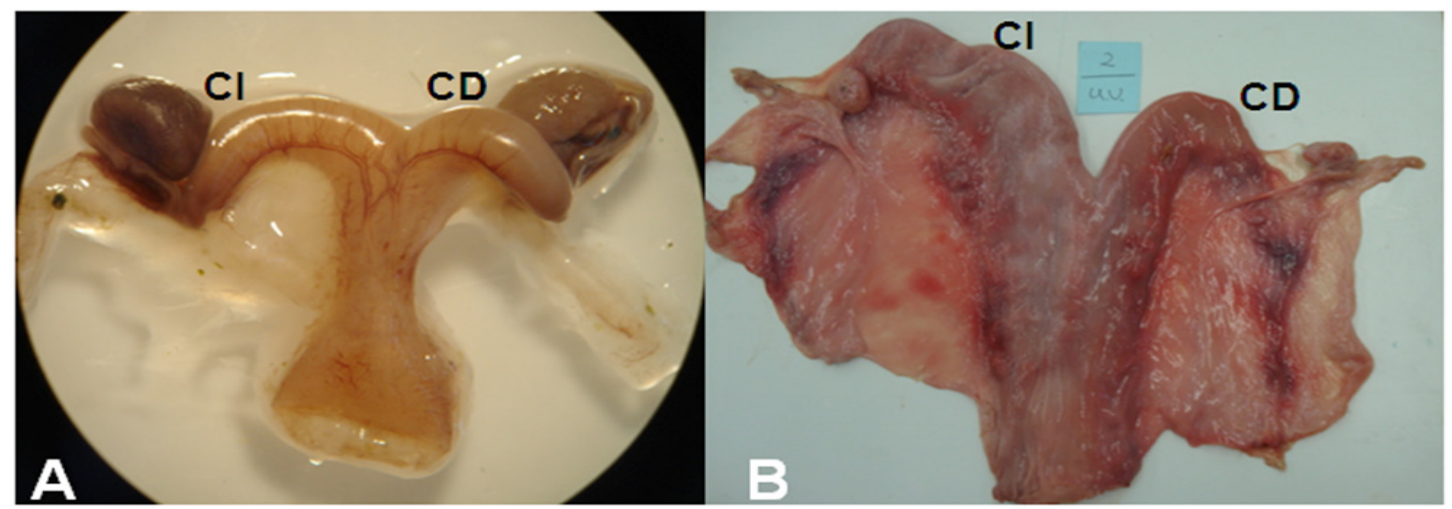

Figura 4: Características macroscópicas del útero no gestante. A: Útero de feto de alpaca de primer tercio de gestación. B: Útero de alpaca adulta. $\mathrm{CI}=$ Cuerno uterino izquierdo; $\mathrm{CD}=$ Cuerno uterino derecho.

El estudio anatómico de los úteros en adultos y fetos, evidenció que los cuernos uterinos al extenderse adoptan una distribución cónica, con un extremo angosto relacionado al oviducto y un extremo ancho relacionado al cuerpo del útero. Esta característica difiere con otros estudios que describen los cuernos uterinos como cilíndricos (1). Sin embargo, coinciden en el hecho que los cuernos adquieren una distribución espiralada o sufren una ligera torsión. La distribución uterina es semejante a la del útero no gestante de la yegua, cerda, vaca y oveja (4) diferenciándose por la posición final de los cuernos uterinos hacia lateral.

El estudio morfométrico realizado para evaluar los cuernos uterinos, mostró diferencia significativa a favor del tamaño del cuerno uterino izquierdo, tanto en la alpaca, como en la llama (Tabla 1). Al evaluar la relación existente entre el tamaño del cuerno uterino derecho y el cuerno uterino izquierdo en la alpaca, esta fue de 0.79 (aproximadamente $80 \%$ ), este valor varía según el estado fisiológico del órgano, siendo mayor al momento en que los ovarios presentan cuerpo lúteo. En la llama, la razón de las medidas es mayor $(0.85$ para la longitud y 0.87 para el ancho) (Tabla 2).

Los resultados obtenidos sobre la morfometría de los cuernos uterinos adultos y fetales tanto en la llama como en la alpaca, son coincidentes con lo descrito por otros autores $(9,8,10)$. Estos resultados demuestran que el mayor tamaño del cuerno uterino no es producto del mayor porcentaje de gestaciones a este nivel sino que se trata de una característica anatómica manifestada ya desde la vida fetal.

\section{CONCLUSIONES}

- La forma, la posición y la distribución de los tractos genitales de los fetos de llama y alpaca son similares al de la alpaca y llama adulta.

- En elúltimo mes de la gestación, para ambas especies el cuerno uterino izquierdo es significativamente de mayor tamaño en relación al cuerno uterino derecho.

- Se concluye que la media entre las razones del largo y ancho de los cuernos uterinos de la alpaca es de 0,79 .

\section{Correspondencia}

Galy Mendoza Torres

Correo electrónico: galy.mendoza@upch.pe 


\section{REFERENCIAS BIBLIOGRÁFICAS}

1. Benirschke K. Genetic management.International Zoo Yearbook. 2007; 17: 50-60,

2. Bravo PW, Mayta MM, Ordonez CA. 2000. Growth of the conceptus in alpacas. Am J Vet Res. 2000; 61: 1508-1511.

3. Bravo PW. The reproductive process of South American Camelids. Salt Lake City, Utah, USA.:Segall Printing;2002.p. 100.

4. Del Campo MR, Del Campo CH, Ginther OJ.

Vascular provisions for a local utero-ovarian cross-over pathway in new world camelids. Theriogenology. 1996; 48: 963-981.

5. Gazitúa FJ, Corradini P, Ferrando G, Raggi LA, Parraguez VH. Prediction of gestational age by ultrasonic fetometry in llamas (Lama glama) and alpacas (Lama pacos). Animal Reproduction Science. 2001; 66: 81-92.

6. König HE, Liebich HG. Anatomía de los animales domésticos.2.ed.Madrid:MédicaPanamericana;2005. p. 400.
7. International Committee on Veterinary Gross Anatomical Nomenclature. Nomina anatómica veterinaria. Fifth edition. Columbia, MO, U.S.A.: International Committee on Veterinary Gross Anatomical Nomenclature, 2012.

8. Sato A, Montoya L. Aparato reproductor de la alpaca (Lama pacos). Revista de Camélidos Sudam. 1990; 7: 5-14.

9. Sumar J, Bravo W. Efecto endocrino fisiológico del Receptal en alpacas.Punta Arenas, Chile:Convención Internacional sobre Camélidos Sudamericanos;1981. p.3.

10. Tibary A, Anouassi A. Theriogenology in camelidae. Mina, Abu Dhavi, UAE:Abu Dhabi Printing; 1997.

Recibido: 31/01/2013

Aceptado: 24/06/2013 